\title{
Gestação e Saúde Bucal: Importância do pré-natal odontológico
}

\author{
Pregnancy and Oral Health: Importance of dental prenatal care \\ Embarazo y Salud Bucal: Importancia del cuidado dental prenatal
}

Recebido: 20/01/2021 | Revisado: 23/01/2021 |Aceito: 25/01/2021 | Publicado: 31/01/2021

\author{
Kelly Alves Guimarães \\ ORCID: https://orcid.org/0000-0003-1979-945X \\ Faculdade Patos de Minas, Brasil \\ E-mail: kelly.alves_2008@hotmail.com \\ Gabriela Andrade Sousa \\ ORCID: https://orcid.org/0000-0002-9078-1802 \\ Faculdade Patos de Minas, Brasil \\ E-mail: gabrielaandradesousa1 @ outlook.com \\ Marcelo Dias Moreira de Assis Costa \\ ORCID: https://orcid.org/0000-0001-9148-3674 \\ Universidade Federal de Uberlândia, Brasil \\ E-mail: marcelodmac@yahoo.com.br \\ Cláudia Maria de Oliveira Andrade \\ ORCID: https://orcid.org/0000-0003-4529-8106 \\ Faculdade Patos de Minas, Brasil \\ E-mail: claudia.andrade@faculdadepatosdeminas.edu.br \\ Lia Dietrich \\ ORCID: https://orcid.org/0000-0001-7887-8591 \\ Clínica privada, Brasil \\ E-mail: lia_dietrich@yahoo.com.br
}

\begin{abstract}
Resumo
O presente trabalho tem como objetivo apresentar os problemas odontológicos comuns durante a gravidez, bem como o manejo odontológico nesse período. Este estudo consiste de uma revisão bibliográfica qualitativa, tendo como fonte de dados escolhidos para pesquisa as bases Scielo, BVS, e Pubmed. Apresentando uma proposta seletiva para eleição dos estudos escolhidos para este trabalho, onde os critérios de seleção foram: pesquisa de artigos de 2010 a 2020 , língua portuguesa, inglesa, e espanhola, que abordam descritores como e/ou relacionados a: gestantes, saúde bucal, cuidado pré-natal, oral health, pregnancy, pregnant women, primary health care, el embarazo, atención odontológica. O pré-natal odontológico deve ser iniciado assim que descoberto a gravidez, contudo, o ideal seria a ocorrência de um planejamento prévio à gestação, para que ocorresse a adequação e promoção da saúde bucal, visto que, resultados adversos são observados em gestações que a mãe se encontra em péssimas condições bucais, onde, a prevenção/tratamento de problemas bucais antes do início gestacional seria sinônimo de uma gestação ainda mais saudável, tanto para a mãe como para o filho. O cirurgião-dentista observaria todo o estado de saúde bucal da gestante, o que possibilita o impedimento de agravos de problemas bucais instalados, e a prevenção de novas alterações.
\end{abstract}

Palavras-chave: Gestantes; Saúde bucal; Cuidado pré-natal.

\begin{abstract}
This paper aims to present common dental problems during pregnancy, as well as dental management during this period. This study consists of a qualitative bibliographic review, using Scielo, BVS, and Pubmed as the source of data chosen for research. Presenting a selective proposal for the election of the studies chosen for this work, where the selection criteria were: research of articles from 2010 to 2020, Portuguese, English, and Spanish, which address descriptors such as and / or related to: pregnant women, oral health, prenatal care, oral health, pregnancy, pregnant women, primary health care, el embarazo, odontológica. Dental prenatal care should start as soon as the pregnancy is discovered, however, the ideal would be the occurrence of planning prior to pregnancy, so that the adequacy and promotion of oral health would occur, since adverse results are observed in pregnancies that mother is in terrible oral conditions, where the prevention / treatment of oral problems before the start of pregnancy would be synonymous with an even healthier pregnancy, both for the mother and the child. The dental surgeon would observe the entire state of oral health of the pregnant woman, which makes it possible to prevent aggravations of installed oral problems, and to prevent new changes.
\end{abstract}

Keywords: Oral health; Pregnant women; Primary health care. 


\begin{abstract}
Resumen
Este artículo tiene como objetivo presentar los problemas dentales comunes durante el embarazo, así como el manejo dental durante este período. Este estudio consiste en una revisión bibliográfica cualitativa, utilizando Scielo, BVS y Pubmed como fuente de datos elegida para la investigación. Presentar una propuesta selectiva para la elección de los estudios elegidos para este trabajo, donde los criterios de selección fueron: investigación de artículos de 2010 a 2020 , portugués, inglés y español, que abordan descriptores como y / o relacionados con: gestante, salud bucal, atención prenatal, salud bucal, embarazo, gestante, atención primaria de salud, el embarazo, odontológica. La atención odontológica prenatal debe iniciarse tan pronto como se descubra el embarazo, sin embargo, lo ideal sería la ocurrencia de planificación previa al embarazo, para que se produjera la adecuación y promoción de la salud bucal, ya que se observan resultados adversos en embarazos que La madre se encuentra en pésimas condiciones bucales, donde la prevención / tratamiento de los problemas bucales antes del inicio del embarazo sería sinónimo de un embarazo aún más saludable, tanto para la madre como para el niño. El cirujano dentista observaría todo el estado de salud bucal de la gestante, lo que permite prevenir agravamientos de los problemas bucales instalados y prevenir nuevos câmbios.
\end{abstract}

Palabras clave: Embarazo; Atención odontológica; Salud bucal.

\title{
1. Introdução
}

Hoje em dia, sabe-se da importância da saúde bucal e de como esta se faz essencial para a saúde geral dos indivíduos, e, quando na sua ausência, interfere negativamente na qualidade de vida das pessoas (Concha Sánchez, Almario Barrera, \& Pabón Ordoñez, 2020; Pereira, 2010).

A Odontologia busca cada vez mais evoluir e tratar o ser humano como um todo, não evidenciando apenas cuidados em boca e nos dentes, mas o estado de saúde geral do paciente, pois, doenças bucais pré-existentes, como, principalmente a doença periodontal, apresentam microorganismos gram-negativos, semelhantes a microorganismos encontrados em diversas infecções crônicas e respiratórias (Lima, Saliba, Garbin, Fernandes, \& Garbin, 2011).

Muito discutido na literatura, a associação entre a saúde bucal e seu impacto sobre a saúde sistêmica, evidencia a necessidade de, quando a procura por algum tipo de atendimento, que o indivíduo consiga ter uma avaliação de forma integral e multiprofissional (Pacheco et al., 2020; Saliba, Custódio, Saliba, \& Moimaz, 2019).

Nesse contexto, é claro que a saúde bucal é imprescindível na vida das pessoas, e adquire uma proporção especial na mulher durante a gravidez, no pós-parto, e também no bebê (Concha Sánchez et al., 2020). A gestação é um período em que os cuidados com a saúde bucal devem ser mais rigorosos, visto que, a atenção odontológica é frequentemente negligenciada, e isso pode influenciar na saúde do bebê (Concha Sánchez et al., 2020; Garbin, Sumida, Santos, Chehoud, \& Moimaz, 2011; Konzen Júnior, Marmitt, \& Cesar, 2019; Pacheco et al., 2020).

Dentro do ciclo da atenção à saúde da gestante, a atenção ao pré-natal compreende grandes funções, como a promoção da saúde, o rastreamento e diagnóstico de doenças, assim também como a prevenção de enfermidades, dessa maneira, os cuidados odontológicos pré-natais, promovem a saúde materno-infantil, uma vez que, mulheres grávidas caracterizam um grupo de risco para doenças bucais devido às suas alterações físicas, hormonais, biológicas e comportamentais, além da elevada prevalência de cárie e doenças periodontais na gestação (Saliba et al., 2019). Existem ainda indicações de que patologias bucais, como a doença periodontal, se relacionadas a precárias condições de vida, pode influenciar a ocorrência de desfechos desfavoráveis, como recém-nascidos prematuros e de baixo peso (Almeida, 2014; Bastos, Silva, Cardoso, Farias, \& Falcão, 2014; Guidolini Martinelli, Belotti, Martins Poletto, Theodoro dos Santos Neto, \& Emmerich Oliveira, 2020; Konzen Júnior et al., 2019; Moimaz, Rovida, Garbin, Santos, \& Saliba, 2015; Pacheco et al., 2020; Saliba et al., 2019).

O atendimento odontológico durante a gestação, pode impulsionar hábitos saudáveis, facilitar a amamentação e consequente estimular o crescimento e desenvolvimento orofacial do bebê (Saliba et al., 2019). Nesta fase, torna-se favorável abordagens para a prática de promoção de saúde, pois a gestante se encontra mais receptiva a adquirir novas informações relacionadas a sua saúde e a do seu bebê, além de poder se tornar agente multiplicadora de hábitos saudáveis também a sua família. Este pode ser o momento mais adequado para mãe analisar como ela percebe sua condição de saúde bucal, para 
concretizar sua importância e real necessidade (Almeida, 2014; Costa, 2014; Lessa, 2013; Martins, Pinheiro, Arantes, Nascimento, \& Santos Júnior, 2013; Mattos, \& Davoglio, 2015; Pacheco et al, 2020; Reis et al., 2010; Silva et al., 2020).

Porém, o atendimento a gestantes, ainda é um assunto bastante controverso, por haver incertezas, tanto da parte das gestantes, que normalmente não procuram o atendimento pela desinformação da sua importância, ou por muitas vezes acreditarem que esta possa implicar em ameaça tanto para sua saúde, como para a de seu filho. O cirurgião-dentista, que por muitas vezes não se sentem seguros em atende-las, consideram adiar ou ainda não realizar procedimentos clínicos no período gestacional (Costa, 2014). Tais receios das gestantes, são provocados por encaminhamentos empíricos, e crenças populares, como riscos da anestesia, hemorragias, a crença de que, independentemente dos cuidados odontológicos com os dentes, eles tendem a se acabar mais frágeis e sujeitos a cárie pela perda de cálcio utilizado para a formação de ossos e dentes da criança em desenvolvimento, a falta de informações (muitas vezes acreditam que a dor de dente estaria associada à condição da gravidez), como também, medo da dor (Almeida, 2014; Cardoso, 2010; Costa, 2014; Garbin et al., 2011; Lessa, 2013; Mattos \& Davoglio, 2016; Pacheco et al., 2020; Reis et al., 2010; Rodrigues, Mármora, Carrion, Rego \& Pospich, 2017; Sá de Lira, Silva, Caetano, Araújo Junior \& Portela, 2019; Silva et al., 2020).

A forma como o acesso a Unidade Básica de Saúde ocorre, também pode interferir na forma de utilização do serviço, e na intensificação da utilização do pré-natal na rede pública, sendo assim, várias ações são realizadas para melhorar o atendimento a gestante. A Política Nacional de Saúde Bucal, que tem por objetivo, ampliar o acesso e ultrapassar o modelo biomédico centrado apenas na doença, propõe a implantação de ações coletivas e atendimento individual, ou seja, no momento em que a mulher inicia o acompanhamento da gestação, ela deverá passar por uma consulta odontológica e também receber informações sobre higiene bucal e alimentação, assim como receber uma avaliação da sua cavidade bucal (Gonçalves et al., 2020; Guidolini Martinelli et al., 2020). Além disso, é válido compreender o perfil das gestantes que procuram o atendimento odontológico, para facilitar sua assistência, a fim de promover uma gestação e mais saudável (Guidolini Martinelli et al., 2020). É importante ressaltar que os profissionais atuam com um papel importante na desconstrução de percepções incorretas sobre o atendimento odontológico durante a gestação, sendo que uma conversa agradável, calma e tranquila durante as consultas pode amplificar o comprometimento das gestante com a saúde bucal (Nunes Neto \& Frutuoso, 2018).

Outros fatores parecem estar relacionados a não utilização de serviços odontológicos por gestantes, entre eles, o nível socioeconômico, falta de tempo, desinteresse, onde, em estudo realizado por Bressane et al. (2011) a maioria das gestantes afirmaram a necessidade de tratamento durante entrevista, no entanto, nenhuma das gestantes buscou atendimento odontológico durante a gravidez; a necessidade de repouso devido à hipertensão, impossibilitando o deslocamento para as consultas (Pacheco et al., 2020). Em relação a escolaridade, observou-se que independentemente do nível educacional da mãe, a maioria recusa-se ao tratamento odontológico durante a gravidez, o que evidencia que a falta de informação sobre o assunto não é restrito à gestantes com um menor nível educacional (Almeida, 2014; Pacheco et al., 2020; Silva et al., 2020).

O atendimento odontológico durante o período da gravidez é geralmente seguro, previne complicações e também melhora a qualidade de vida da mulher grávida, enquanto reduz patógenos orais e o risco de transmissão da mãe para os filhos (Nunes Neto \& Frutuoso, 2018). O Ministério da Saúde reconheceu que a saúde bucal da gestante está relacionada com a saúde geral e pode influenciar a saúde bucal e geral do bebê também, assim, o atendimento multiprofissional e transdisciplinar à saúde da gestante, é de grande importância, incluindo médicos pediatras, obstetras, ginecologistas, enfermeiros, cirurgiõesdentistas, entre outros profissionais da saúde (Nunes Neto \& Frutuoso, 2018; Saliba et al., 2019). Diante do exposto, este trabalho tem como objetivo apresentar os problemas odontológicos comuns durante a gravidez, bem como deve ser o atendimento odontológico nesse período e os principais cuidados. 


\section{Metodologia}

Este estudo consiste de uma revisão bibliográfica qualitativa, tendo como fonte de dados escolhidos para pesquisa as bases Scielo, BVS, e Pubmed. Apresentando uma proposta seletiva para eleição dos estudos escolhidos para este trabalho, onde os critérios de seleção são: pesquisa de artigos de 2010 a 2020, língua portuguesa, inglesa, e espanhola, que abordam descritores como e/ou relacionados a: gestantes, saúde bucal, cuidado pré-natal, oral health, pregnancy, pregnant women, primary health care, el embarazo, atención odontológica.

\section{Revisão de Literatura}

\subsection{Alteraçães hormonais e fisiológicas do corpo da mulher no período da gestação}

O ciclo gestacional é uma fase constantemente marcada por várias alterações, tanto físicas, biológicas, hormonais, anatômicas e psicológicas (Saliba et al., 2019; Silva et al, 2020). Dentro da normalidade, a gestação dura por volta de 9 meses, mais especificamente 40 semanas, períodos que são divididos por trimestres, onde cada trimestre terá alterações fisiológicas importantes que devem ser conhecidas pelo cirurgião-dentista (Catão, Gomes, Rodrigues \& Soares, 2015; Marla, Srii, Roy \& Ajmera, 2018).

Na fase inicial da gestação (primeiro trimestre) a existência de náuseas e vômitos é comum na maioria das mulheres, podendo persistir por toda gestação. Estes são ocasionados pelo desequilíbrio da ação metabólica, provocado pelo aumento das taxas hormonais, inclusive a progesterona (Reis et al., 2010). Entre a $25^{\circ}$ e $36^{\circ}$ semana de gestação, observa-se na mulher uma discreta elevação da pressão arterial que pode vir acompanhada de taquicardia, sendo uma condição considerada normal, onde, pode-se ocorrer um diagnóstico de hipertensão durante a gravidez quando os níveis da pressão arterial encontram-se maiores que 140/90 mmHg. Este aumento da pressão pode ser classificado como pré-eclâmpsia, quando a hipertensão arterial aparece depois da $20^{\circ}$ semana de gestação ligada a proteinúria (Aleixo, Moura, Almeida, Silva \& Moreira, 2016; Jacob, Santos, Lopes \& Shimo, 2020; Reis et al., 2010). Em relação ao sistema respiratório, alterações fisiológicas como o aumento no consumo de oxigênio de cerca de 15 a $20 \%$ e a capacidade de reserva funcional diminui pela compressão do diafragma pelo constante aumento do tamanho do útero, eleva o risco de dispneia e apneia na posição supina (Aleixo et al. 2016; Bastos et al., 2014; Lessa, 2013; Reis et al., 2010).

O útero gravídico, já no terceiro e último trimestre, causará compressão da artéria aorta e veia cava, aumentando a chance da mulher apresentar hipotensão ortostática, quando em posição supina (Bastos et al., 2014; Lee, \& Shin, 2017; Reis et al., 2010). Durante a evolução da gravidez, o aumento do volume uterino, comprime o estômago, e consequentemente leva ao aumento do número de refeições em menores quantidades. Esse aumento da frequência de alimentação associado a falta de uma boa higiene bucal, eleva a probabilidade do desenvolvimento da doença cárie na gestante. $\mathrm{O}$ aumento do volume uterino leva ao ganho de peso e frequente necessidade de urinar (Almeida, 2014; Bastos et al., 2014; Konzen Júnior et al., 2019; Moimaz et al., 2015; Reis et al., 2010).

A gravidez é um período transitório, e a variedade de mudanças ocorridas dá-se pela influência contínua de hormônios, como estrogênio e progesterona, onde, a elevação destes vai se apresentar de variados sinais e sintomas, que podem modificar o estado de saúde geral, interferir na cavidade bucal, exacerbando patologias como as doenças periodontais (Marla et al., 2018; Silva et al., 2020).

\subsection{Alterações bucais}

A boca, como parte integrante do organismo, também pode passar por modificações na gravidez (Reis et al., 2010). Com as alterações hormonais, observa-se um aumento na vascularização periférica, aumento do fluxo de fluido gengival, aumento da síntese de prostaglandinas e maior permeabilidade dos vasos sanguíneos da gengiva pelo aumento de progesterona, 
o que pode levar ao início de processos inflamatórios, com a presença do biofilme dental, ou ao aumento da intensidade dos mesmos (Aleixo et al., 2016; Bastos et al., 2014; Catão et al., 2015; Martins et al., 2013; Oliveira, Lopes, Santos, \& Magalhães, 2014; Reis et al., 2010).

Nessa fase, juntamente com a maior vascularização do tecido periodontal, onde Rosell FL e colaboradores (1999) correlaciona tal acontecimento pela presença de biofilme, a elevação dos níveis de estrogênio e progesterona, carências nutricionais e ainda o estado transitório de imunodepressão, ocorre também, uma hipersecreção das glândulas salivares, especialmente nos meses iniciais da gestação, onde este fenômeno (sialorreia) não tem ainda uma causa definida, podendo durar a gestação inteira (Lessa, 2013; Mattos, \& Davoglio, 2015; Reis et al., 2010).

Diversas são as manifestações de alterações bucais que podem ser encontradas na gravidez, onde as mais comuns são a doença periodontal e a cárie dentária, mas vale salientar que o período da gravidez não é um fator determinante para o surgimento de tais ocorrências, e podem ser evitáveis (Almeida, 2014; Catão et al., 2015; Moimaz et al., 2015; Oliveira et al., 2014; Reis et al., 2010; Rocha et al., 2018; Saliba et al., 2019).

\subsubsection{Principais doenças bucais predisponentes durante a gestação}

Várias são as alterações bucais que podem ocorrer no período da gestação, dentre as mais comuns, temos as doenças periodontais e a cárie dentária (Reis et al., 2010). É importante dizer que as alterações hormonais refletem na fisiologia oral, e que alteram o equilíbrio normal da boca, porém, a gravidez não é um fator determinante para o aparecimento de tais manifestações, o que ocorre, é que essas alterações se já preexistentes, tendem a se agravar, principalmente se houver descuido da saúde bucal. Então, os cuidados da saúde bucal durante o período gestacional são de grande importância, por mais que, a maioria da população não tenha conhecimento das alterações relativas a esse período (Catão et al., 2015; Garbin et al., 2011; Lessa, 2013; Lopes, Pessoa \& Macêdo, 2018; Martins et al., 2013; Reis et al., 2010; Sousa, Cagnani, Barros, Zanin \& Flório, 2016).

A cárie dentária é uma doença multifatorial, e existem evidências do aumento da incidência de cáries em mulheres grávidas, sendo que o principal fator para tal aparecimento, é a presença do biofilme dental, que também predispõem o aparecimento da doença periodontal (Massoni et al., 2015). O estado das lesões cariosas durante a gestação, pode ser sugestionado por fatores comportamentais, visto que, ocorre uma mudança nos padrões alimentares, onde a gestante passa a ter um consumo constante de alimentos cariogênicos, o que leva a diminuição do $\mathrm{pH}$ oral, favorecendo o aparecimento da lesão (Costa et al., 2017; Lessa, 2013; Marla et al., 2018; Massoni et al., 2015; Oliveira et al., 2014).

Os episódios de vômitos, também levam a diminuição do pH bucal e capacidade tampão saliva, que podem interferir no aparecimento da cárie dentária (Costa et al., 2017). A incidência da cárie é ainda ampliada, pela questão que, os enjoos matinais, levam também a erosão das superfícies dentárias, além de atrapalhar os hábitos de higiene oral de rotina (Bastos et al., 2014; Lessa, 2013; Marla et al., 2018). A escovação dentária é imprescindível para a prevenção da doença cárie, pois evita o processo de desmineralização do esmalte dentário e desorganiza a placa bacteriana (Massoni et al., 2015).

A doença periodontal é uma infecção bacteriana, advinda da associação do biofilme dental não removido com os tecidos periodontais, e tal infecção, além de levar a problemas na cavidade oral (como a gengivite e periodontite), pode levar ao desenvolvimento de problemas sistêmicos durante a gestação, como um parto pré-maturo (Catão et al., 2015). A inflamação gengival, observada no período da gestação, normalmente recebe o nome de gengivite gravídica e é intensificada pela produção dos hormônios sexuais femininos, que provocam alta produção de prostaglandinas (principalmente a progesterona) que aumentam o processo inflamatório na região (Aleixo et al., 2016; Saínz, Paula, Acosta, \& Trujillo, 2019). Geralmente, a gengivite gravídica inicia-se por volta do $3^{\circ}$ a $4^{\circ}$ mês de gestação e atinge sua maior severidade por volta do $8^{\circ}$ mês, nesse período, ocorre um desenvolvimento de periodontopatógenos, como a Prevotella intermedia, e ao decorrer da gravidez a 
microbiota subgengival torna-se cada vez mais anaeróbia (Aleixo et al., 2016; Bastos et al., 2014; Reis et al., 2010; Saínz et al., 2019; Cardoso et al., 2021).

Ao longo da gravidez, os tecidos gengivais são mais susceptíveis a inflamação, porém, tal inflamação não ocorrerá em todas as mulheres, visto que, a uma forte influência da saúde bucal da gestante antes do período da gestação, além da alimentação e os hábitos de higiene oral. Vale ressaltar, que as características inflamatórias podem ser controladas com um correto controle do biofilme (Aleixo et al., 2016; Saínz et al., 2019) A mobilidade dentária também pode ser observada durante a gravidez e se dá ao agravamento do estado periodontal da gestante, por distúrbios na lâmina dura, que surgem pela microflora mais anaeróbia e o aumento de mediadores inflamatórias na região gengival (Marla et al., 2018).

Também induzido pelas alterações hormonais decorrentes da gravidez, outra alteração frequente encontrada em gestantes é o chamado granuloma piogênico (Marla et al., 2018). Este é considerado um tumor reativo, benigno, de maior prevalência em mulheres e encontrado em cerca de 5\% das mulheres grávidas, daí o nome bastante conhecido como “granuloma gravídico". Sua etiologia é decorrente de traumas crônicos e por longa duração, como raízes residuais, dentes cariados e a presença de biofilme que culminam em tártaro dentário subgengival (Marla et al., 2018; Sá de Lira et al., 2019). Clinicamente apresenta-se como um aumento de volume exofídico, de coloração rosa ao vermelho intenso, podendo ser de base séssil ou pediculada, com a superfície lisa ou lobulada e ainda ulcerada, geralmente nodular e bem circunscrito, com uma prevalência maior na maxila na região gengival, podendo surgir ainda em língua, mucosa, lábios e menos frequente em palato duro (Sá de Lira et al., 2019). Dependendo do volume, espera-se a regressão espontânea após o período da gravidez com a normalização dos níveis hormonais (Bastos et al., 2014; Marla et al., 2018; Oliveira et al., 2014; Sá de Lira et al., 2019).

\subsection{Métodos de prevenção de alterações bucais na gestação}

Quando falamos em cuidados com a saúde bucal durante o período gestacional, temos como objetivo, o estabelecimento de uma condição saudável da mãe, e também do seu bebê, além disto, a inclusão de hábitos saudáveis que podem favorecer a prevenção de doenças bucais no início da infância da criança e que vão refletir na saúde bucal do indivíduo durante toda sua vida (Garbin et al., 2011; Lessa, 2013; Moimaz et al., 2015; Silva et al., 2020).

A promoção de saúde, é uma estratégia para buscar a melhora na qualidade de vida da população, sendo assim, a Conferência Internacional sobre Promoção de Saúde, que foi realizada em Ottawa, no Canadá, em 1986 determinou uma série de princípios éticos e políticos, definindo campos de ação, e de acordo com este documento, promoção de saúde é “o processo de capacitação da comunidade para atuar na melhoria da sua qualidade de vida e saúde, incluindo maior participação no controle desse processo" (Lessa, 2013).

O cirurgião-dentista, tem a competência de trabalhar na Equipe de Saúde da Família (ESF), de forma a "Realizar a atenção integral em saúde [...] a grupos específicos, de acordo com um planejamento local, com resolubilidade” (Mattos, \& Davoglio, 2015). E não só o cirurgião-dentista, mas toda a sua equipe auxiliar, técnicos de saúde bucal e auxiliares estão envolvidos nessa estratégia, pois é competência do profissional auxiliar em saúde bucal "Realizar ações de promoção e prevenção em saúde bucal [...] mediante planejamento local e protocolos de atenção à saúde” (Mattos, \& Davoglio, 2015; Reis et al., 2010).

A literatura descreve o desconhecimento das gestantes quanto as reais razões dos problemas bucais e o processo saúde-doença, e também na autopercepção de saúde bucal, pois o comportamento das pessoas em relação a sua saúde oral, depende da forma de como esta é percebida. Diante disto, métodos educativos e a promoção de saúde se fazem eficazes para a melhora do conhecimento e interesse das gestantes sobre sua saúde bucal, e de como tais cuidados podem ser benéficos a elas (Mattos, \& Davoglio, 2015; Moimaz et al., 2015).

Com isso, o comportamento da gestante em relação a sua saúde bucal deve ser conhecido, compreender o que pensam 
na sua ótica de vida, seus costumes e cultura, também é importante, para que hábitos e comportamentos em relação a sua saúde bucal sejam reforçados ou modificados, além de quebrar mitos sobre o atendimento odontológico durante a gravidez, fazendo com que as futuras mães entendam que nesse período de suas vidas, ocorrem alterações fisiológicas de modo sistêmico, mas que afetam o sistema estomatognático, e que problemas neste podem afetar sua saúde e de seu bebê, para que, estratégias efetivas de educação em saúde e demais atividades curativas e de prevenção sejam boas ferramentas para o sucesso do prénatal odontológico, e que sejam corretamente planejas e executadas nos programas de atenção à saúde da mulher (Garbin et al., 2011; Lessa, 2013; Mattos, \& Davoglio, 2015; Moimaz et al., 2015, Reis et al., 2010, Silva et al., 2020).

A gestante deve ser sempre apreciada, quando de forma espontânea procura o atendimento odontológico, já que, o baixo acesso a atendimentos pode estar relacionado a vários fatores, e o principal motivo da procura de uma gestante ao Cirurgião-dentista durante a gravidez, é devido a quadros de urgências e dor, o que leva as práticas promotoras de saúde e os cuidados preventivos a serem esquecidos (Mattos, \& Davoglio, 2015; Moimaz et al., 2015; Reis et al., 2010).

Medidas de auto cuidado, como a escovação dentária e uso de fio dental diários, mesmo sendo considerados a principal medida para prevenir problemas bucais no geral, ainda é considerado falho entre gestantes, daí a necessidade de diferentes abordagens sobre as doenças bucais, fazendo-as compreender seus fatores determinantes e a compreensão da saúde bucal fazendo parte da saúde de todo organismo, o que pode favorecer o emprego de medidas preventivas e a procura e/ou valorização dos atendimentos odontológicos (Moimaz et al., 2015).

Quando falamos da modificação de determinados hábitos, como por exemplo da dieta da gestante, para alimentos com menos carboidratos e açucares, que aumentam o risco de desenvolvimento da cárie dentária quando associada a falta de higienização bucal, não basta meramente recomendar a mudança, mas orienta-las sobre como executá-las e fazer com que tomem conhecimento dos benefícios, pois o ponto de partida de qualquer processo de intervenção é constituído na relação entre o ser humano e o conhecimento (Mattos, \& Davoglio, 2015).

Então, o Ministério da Saúde recomenda que ações educativas e preventivas sejam iniciadas juntamente com o início do pré-natal, e que este seja organizado para atender as reais necessidades da gestante, buscando que esta tenha uma gestação saudável, uma boa assistência e incentivo ao auto cuidado (Lessa, 2013).

\subsection{Cuidados no tratamento odontológico de gestantes}

As gestantes durante o tratamento odontológico, de fato, requerem cuidados especiais e abordagem diferenciada devido às alterações que são inerentes ao período gestacional, mas isso não significa que, nenhuma necessidade de atendimento odontológico vindo de pacientes grávidas deve ser negligenciada por medo de expor a gestação em risco (Cardoso, 2010; Oliveira et al., 2014).

Esta condição, ao contrário do que se pensa, não impede a maioria dos tratamentos odontológicos realizados em rotina, como por exemplo, instruções de higiene oral, avaliação da condição bucal da gestante, profilaxias (limpezas rotineiras), raspagens e alisamentos radiculares, a aplicação tópica de flúor (quando há necessidade), polimentos de restaurações, remoção de cárie e restaurações, visto que, tais cuidados odontológicos são de bem menos risco ao bebê do que por exemplo o aumento de prostaglandinas por causa de um foco infeccioso na cavidade oral, contudo, estudos já demonstram que qualquer tipo de intervenção odontológica pode e deve ser realizada no período gestacional, pois já sabemos que a manutenção da saúde bucal é de grande importância nesse período (Almeida, 2014; Camargo et al., 2014; Cardoso, 2010; Moimaz et al., 2015; Oliveira et al., 2014; Piccirillo, 2012).

O primeiro trimestre da gestação, é onde ocorre todos os fenômenos de desenvolvimento do embrião, que progride para à formação do feto, sendo então, um momento crítico, pois qualquer tipo de complicação, pode contribuir para o aparecimento de efeitos teratogênicos no feto em desenvolvimento, e ainda, ao aborto (Marla et al., 2018; Piccirillo, 2012). E 
falando em saúde bucal, geralmente a recomendação é que as pacientes gestantes passem apenas por avaliações, adequações do meio bucal e que sejam instruídas quanto à manutenção da sua saúde oral, não passando por nenhum procedimento sempre que possível. Pois, em um eventual problema na gestação ou no bebê recém-nascido, a busca por fatores determinantes acontece, e devido a todos os preconceitos já estabelecidos na sociedade diante do tratamento odontológico na gestação, a "culpa" pode se estabelecer diante de procedimentos realizados no consultório. (Almeida, 2014; Moimaz, Rós, Saliba \& Garbim, 2017; Piccirillo, 2012; Rodrigues et al., 2017; Silva et al., 2020)

Entretanto, é unânime entre autores, que os tratamentos devem ser preferencialmente realizados durante o $2^{\circ}$ trimestre da gestação, pois este se caracteriza como o espaço de tempo de maior estabilidade da gravidez, sendo o $3^{\circ}$ trimestre também, seguro para procedimentos eletivos, contudo, como corresponde a fase de término da gestação, causa maiores desconfortos à mãe (Marla et al., 2018; Oliveira et al., 2014; Piccirillo, 2012; Rodrigues et al., 2017).

A prevenção sempre é priorizada, mas na presença de urgências e emergências, como dor e infecções, estas devem ser tratadas em qualquer período gestacional, por meio de endodontias, extrações e drenagens, valendo ressaltar que diante de qualquer tratamento que a paciente grávida necessite, devem ser sempre realizados posteriormente à uma boa anamnese, observando as condições gerais da paciente, daí a relevância de um bom entrosamento entre todos os profissionais que fazem o acompanhamento do pré-natal, para que toda a equipe decida a melhor forma, e os melhores momentos para realização das intervenções essenciais, não postergando os tratamentos, pois isto pode causar mais danos com o desenvolvimento do problema, do que com o tratamento quando feito um diagnóstico (Almeida, 2014; Bastos et al., 2014; Camargo et al., 2014; Cardoso, 2010; Lopes et al., 2018; Martins et al., 2013; Moimaz et al., 2015; Moimaz et al., 2017; Oliveira et al., 2014; Piccirillo, 2012).

As consultas odontológicas, muitas vezes podem ser motivo de grande estresse e ansiedade para as gestantes. Diante de tal quadro, algumas recomendações podem ser convenientes, como: a realização de consultas curtas, no período da segunda metade da manhã (quando enjoos matinais tem menor frequência), monitoramento dos sinais vitais (como frequência cardíaca, e pressão da paciente) a observação do nível de glicose no sangue, a optação por procedimentos mais conservadores e menos invasivos, a adequação do posicionamento da cadeira odontológica, com o encosto da cadeira ligeiramente elevado, ou a paciente em posição de decúbito lateral esquerdo, evitando prolongadas posições supinas (principalmente no terceiro trimestre, pois o aumento da pressão uterina pode provocar náuseas, tonturas e síndrome hipotensiva supina), e na presença de vômitos durante o atendimento, este deve ser suspenso imediatamente (Camargo et al., 2014; Lopes et al., 2018; Marla et al., 2018; Moimaz et al., 2017; Oliveira et al., 2014; Piccirillo, 2012; Rodrigues et al., 2017; Cardoso et al., 2021).

Aos procedimentos que requerem o uso de anestésicos locais, estes de maneira geral, podem ser usados com segurança na gestante (Almeida, 2014; Camargo et al., 2014; Cardoso, 2010; Piccirillo, 2012). Mas algumas ressalvas como, que a Prilocaína e Articaína devem ser evitadas pelo risco de ocorrer metemoglobinemia. O vasoconstritor Felipressina também deve ser evitada pelo risco de diminuir a circulação placentária, além de dificultar a fixação do embrião ao útero e ainda induzir contrações uterinas, mesmo os efeitos adversos sendo observados apenas na administração de altas doses do medicamento, o que não é observado em procedimentos odontológicos de rotina, devem ser de conhecimento do cirurgiãodentista (Bastos et al., 2014; Cardoso, 2010; Saliba et al., 2019). Diante disto, o anestésico Lidocaína a 2\% é o mais indicado para procedimentos odontológicos em gestantes (Almeida, 2014; Bastos et al., 2014; Cardoso, 2010; Martins et al, 2013; Rodrigues et al., 2017). E em relação ao vaso constritor, se não tiver nenhuma contra indicação sistêmica, estes devem sim ser usados, pois prolongam o efeito anestésico, aumentando o tempo de trabalho, dando mais conforto para a paciente, diminuem a quantidade de doses e a toxicidade, onde o mais indicado para gestantes é epinefrina/adrenalina 1:100.000 juntamente com a Lidocaína 2\%, no máximo 2 tubetes por sessão (Bastos et al, 2014; Cardoso, 2010; Martins et al., 2013; Moimaz et al., 2017; Piccirillo, 2012, Rodrigues et al., 2017; Saliba et al., 2019). 
Em relação ao uso de medicamentos prescritos, o ideal é que em uma gravidez nenhum tipo de medicamento fosse ingerido, contudo, mesmo os indicados, devem ser utilizados em casos de real necessidade, pois grande maioria dos medicamentos utilizados passam da mãe para o feto por meio da placenta (Camargo et al., 2014; Lee \& Shin, 2017). Más formações fetais são geralmente causadas pelo uso de medicamentos no primeiro trimestre da gestação. A exposição a medicamentos no segundo e terceiro trimestres estão relacionados a efeitos de funcionamento dos órgãos do bebê (Cardoso, 2010). Todas as drogas possuem concentrações que não devem ser ultrapassadas na sua utilização no período gestacional, o que tem de ser de conhecimento do cirurgião-dentista para evitar possíveis anormalidades no feto, além também do profissional conhecer os medicamentos que prescrevem para suas pacientes gestantes (Camargo et al., 2014; Cardoso, 2010).

O uso de medicação na fase gestacional deve ser avaliado meticulosamente, em relação a antibióticos, a penicilina tem sido considerada para primeira escolha nos tratamentos de infecções bucais, enquanto que ao uso de anti-inflamatórios, estes devem ser usados com grande cautela, pois são contra-indicados durante a gravidez, principalmente no último trimestre da gestação, pois os anti-inflamatórios não esteroidais (AINE's) podem provocar inércia uterina e/ou um fechamento prematuro no canal arterial do feto, hipertensão arterial pulmonar, prolongar o trabalho de parto, e se associados ao uso de Aspirina podem interferir na agregação plaquetária, predispondo por exemplo, hemorragias em casos de cirurgias odontológicas (Bastos et al., 2014; Camargo et al., 2014; Martins et al., 2013; Saliba et al., 2019). Sobre o uso de analgésicos, o Paracetamol seguido da Dipirona sódica são considerados seguros para uso contra dor orofacial na gestação (Bastos et al., 2014; Martins et al., 2013; Saliba et al., 2019).

Os exames radiográficos não precisam ser adiados durante a gestação, podendo ser realizados sempre que necessário, pois sabe-se que as doses de radiação utilizadas nas tomadas radiográficas odontológicas são muito baixas para conseguir causar algum tipo de má formação no feto em desenvolvimento (Cardoso, 2010). As gestantes devem ser informadas em relação à segurança de tais exames radiográficas, e além disso, o uso de filmes ultrarrápidos, o uso de colares tireoidianos, não direcionamento da ampola para o abdômen, o maior cuidado para não ocorrer erro da técnica para evitar repetição do exame, e ainda o uso do avental de chumbo, que se encontra cobrindo todo o abdômen da gestante, garantem uma eficaz proteção para a futura mamãe, contudo, devem ser evitadas radiografias de rotina, e que não estejam relacionadas ao local de interesse, e também, durante o primeiro trimestre devem ser indicados em casos de grande necessidade (Almeida, 2014; Bastos et al., 2014; Camargo et al., 2014; Cardoso, 2010; Martins et al., 2013; Moimaz et al., 2017; Piccirillo, 2012).

Diante de tudo, cabe ao profissional cirurgião-dentista e equipe, serem responsáveis por um atendimento seguro e eficaz para a gestante e também para o feto em desenvolvimento, criando principalmente protocolos para prevenção e manutenção da saúde bucal, passando segurança e tranquilidade para as pacientes diante de qualquer tratamento que seja proposto (Oliveira et al., 2014).

\section{Discussão}

O pré-natal, deve ser iniciado assim que descoberto a gravidez, ou seja, de preferência que seja ainda no primeiro trimestre, para que, o estabelecimento precoce de bons e essenciais hábitos de higiene bucal e também orientações sobre a dieta sejam priorizados no decorrer do pré-natal e da gestação (Lopes et al., 2016). Contudo, é importante levantarmos a questão, que o ideal seria que a mulher ao iniciar o seu período gestacional, já estivesse com a sua saúde bucal em dia, favorecendo uma gestação saudável, e que não fosse necessário submeter-se a tratamentos odontológicos neste período, fazendo apenas o acompanhamento para manutenção da sua saúde bucal.

Uma das principais razões para o aparecimento dos problemas bucais durante a gravidez, são as oscilações hormonais (que promovem uma resposta inflamatória que facilita o desencadeamento da doença periodontal na presença do biofilme dental) e também a mudança de dieta pela grávida nesse período, o que favorece às gestantes a ficarem com um risco maior de 
sofrer doenças bucais (Lopes et al., 2016; Teshome, \& Yitayeh, 2016), o que pode vir a desencadear outros problemas de forma sistêmica, onde já exaltamos a importância da saúde bucal na saúde geral, e vice-versa.

Durante o período em que a mulher se encontra grávida, todos os seus cuidados e anseios, são voltados para o seu bebê, onde deveria de certa forma, a gravidez vir a ser planejada, o que favoreceria ainda mais a saúde tanto da mãe, como do seu filho (Reis et al., 2010).

Levando em conta, a importância do cuidado ao binômio mãe-filho, mulheres grávidas consideradas como período gestacional de alto risco, que são classificadas em relação a sua idade, relações com tabagismo, doenças cardíacas, diabetes, hipertensão, obesidade, problemas sanguíneos, e as doenças sexualmente transmissíveis (DST's), estão sujeitas a morbimortalidade na gestação, onde, na maioria das vezes, tais desfechos são preveníveis, quando identificados e acompanhados precocemente (Moimaz et al., 2017).

Entretanto, se uma gravidez, fosse ponderada e planejada, juntamente com toda a equipe de saúde, como médicos, cirurgiões-dentistas, nutricionistas, psicólogos, entre outros, as chances do desenvolvimento saudável da gestação seriam maiores (Lessa, 2013), do que ao começar um tratamento após a descoberta da gravidez, onde já mencionado, o ideal seria o começo do pré-natal ainda no primeiro trimestre, com um acompanhamento mensal da gestante, o que muitas vezes não acontece, pelo descobrimento tardio da gestação/ausência de sinais, ou ainda pela gestante não ter preocupação se está grávida, por não querer/pensar na gravidez no momento, ou por fazer o uso de métodos contraceptivos (Marla et al., 2018; Piccirillo, 2012).

Diante de vários estudos na área, observa-se forte correlação entre a falta de saúde bucal no período gestacional e resultados adversos na gravidez, logo, algumas manifestações como a cárie e a doença periodontal (gengivite e periodontite) são comuns entre gestantes (pelas alterações hormonais 50 a $70 \%$ das mulheres grávidas desenvolvem a gengivite na gestação), e a doença periodontal, que se caracteriza como uma doença de caráter bacteriano (principalmente bactérias gramnegativas) e inflamatório que afeta tanto as estruturas duras quanto moles que sustentam os dentes, que na sua forma mais agressiva, a periodontite, a gengiva se afasta do dente e os tecidos de suporte gengivais são totalmente destruídos, pode ser uma fator de risco para o desenvolvimentos de partos prematuros, e crianças com baixo peso (Gesase et al., 2018; Rosa, Pires, Medeiros, Edelweiss, \& Martínez-Mesa, 2012; Teshome, \& Yitayeh, 2016).

O nascimento prematuro é aquele que se caracteriza como uma idade gestacional inferior a 37 semanas completas, e o baixo peso, aquele que apresenta um peso abaixo dos 2,500kg ao nascimento (Gesase et al., 2018; Rosa et al., 2012). Mulheres grávidas com a doença periodontal em estado ativo, pode vir a sofrer uma indução à cascata de mediadores inflamatórios, induzida por bactérias periodontais, que pode vir a provocar resultados adversos na gravidez, e ainda, tais patógenos periodontais ou subprodutos destes, podem cair na corrente sanguínea chegando até a placenta, e se espalhar pela circulação fetal, o que pode causar abortos espontâneos, ou o parto prematuro (Gesase et al., 2018; Teshome \& Yitayeh, 2016). Tal inflamação/infecção, podem acarretar em alterações na estrutura da placenta, levando ao desenvolvimento de pré-eclâmpsia, comprometimento da nutrição do feto, o que reduz o seu peso, e tal exposição fetal, pode aumentar as taxas de morbimortalidade perinatal (Gesase et al., 2018).

Todos esses contratempos, são passíveis de prevenção, se mulheres que pretendem ter filhos cuidassem regularmente da sua saúde oral, com visitas regulares ao cirurgião-dentista, este que além de promover a saúde bucal para o recebimento da futura condição da mulher, ainda aconselharia a toda importância da manutenção da saúde bucal durante o pré-natal, bem como após esse período, e os cuidados orais com o recém-nascido, e ainda caberia informar sobre desmistificação de todos os tabus referentes a gravidez e a Odontologia, o que facilitaria a aceitação da futura gestante a passar por consultas odontológicas neste período (Silva et al., 2020).

Bem como, das mulheres que não planejaram ou esperavam a gravidez em determinado momento de suas vidas, vale 
ressaltar a importância de sempre se pensar na saúde da boca, como se pensa na saúde do corpo, para que a saúde bucal seja algo de constante importância, visto que, a doença periodontal e seus agravos não afetam apenas mulheres grávidas, mas qualquer indivíduo que não a trate.

\section{Considerações Finais}

O desenvolvimento do presente estudo possibilitou ressaltarmos a importância da presença do pré-natal odontológico na vida da gestante, bem como os aspectos positivos e negativos (quando na sua falta) que este pode influenciar na vida da mulher. O pré-natal odontológico possibilita que o cirurgião-dentista faça observações de todo o estado de saúde bucal da gestante, possibilitando o impedimento de agravos de problemas já instalados na cavidade bucal, bem como o aparecimento de novas alterações. Dada à importância do assunto, faz-se necessário maiores estudos principalmente tendo como foco o conceito de que o ideal é que fossem realizadas avaliações odontológicas preventivas antes da gravidez, o que possibilitaria mais ainda o desenvolvimento de uma gestação saudável, sem preconceitos sobre o atendimento odontológico na gestação, e aumentando o conhecimento das futuras mães sobre higiene bucal, e a relevância desta, tanto na sua vida, como na de seu futuro filho. O desenvolvimento do pré-natal odontológico é uma atividade simples, contudo, representa maior qualidade de vida para o binômio mãe-filho.

\section{Referências}

Aleixo, R., Moura, C., Almeida, F., Silva, H., \& Moreira, K. (2016). Alterações bucais em gestantes - revisão de literatura. Revista Saber Científico, 1(1), 6880. http://revista.saolucas.edu.br/index.php/resc/article/view/655/144.

Almeida, F. M. (2014). Orientação às gestantes com intuito de promoção, educação e prevenção da cárie no psf Dr. Mardome Balduino Rezende. Trabalho de conclusão de curso apresentado ao curso de especialização em Atenção Básica em Saúde da Família, Universidade Federal de Minas Gerais, Uberaba, MG. https://www.nescon.medicina.ufmg.br/biblioteca/imagem/6203.pdf.

Bastos, R. D. S., Silva, B. dos S., Cardoso, J. A., Farias, J. G. de, \& Falcão, G. G. V. C. S. (2014) Desmistificando o atendimento odontológico à gestante: revisão de literatura. Revista Bahiana de Odontologia 5(2), 104-116. https://www.researchgate.net/publication/284566178_DESMISTIFICANDO_O_A TENDIMENTO_ODONTOLOGICO_A_GESTANTE_REVISAO_DE_LITERATURA.

Camargo, M. C., Sakashita, M. S., Ferlin, C. R., Oliveira, D. T. N. de, Bigliazzi, R, \& Bertoz, F. A. (2014). Atendimento e protocolo indicados na odontologia à gestante: revisão de literatura. Revista Odontológica de Araçatuba, 35(2), 55-60. https://apcdaracatuba.com.br/revista/2015/03/TRABALHO\%209.pdf.

Cardoso, L. M. (2010) Atendimento odontológico da gestante na estratégia do programa saúde da família. Trabalho de conclusão de curso apresentado ao curso de especialização em Atenção Básica em Saúde da Família, Universidade Federal de Minas Gerais, Corinto, MG. https://www.nescon.medicina.ufmg.br/biblioteca/imagem/2316.pdf.

Cardoso, L. S., Costa, B. M. de M., Silva, M. S. O. e, Pessoa, T. M., Costa, B. M. de M., \& Trinta, R. R. S. (2021). Conhecimento dos cirurgiões-dentistas sobre atendimento odontológico em gestantes. Research, Society and Development, 10(1), e24510111701. https://doi.org/10.33448/rsd-v10i1.11701

Catão, C. D. de Sá, Gomes, T. de A., Rodrigues, R. Q. F., \& Soares, R. de S. C. (2015). Evaluation of the knowledge of pregnant women about the relationship between oral diseases and pregnancy complications. Revista de Odontologia da UNESP, 44(1), 59-65. https://doi.org/10.1590/1807-2577.1078. https://www.scielo.br/scielo.php?script=sci_arttext\&pid=S1807-25772015000100059.

Concha Sánchez, S., Almario Barrera, A., \& Pabón Ordoñez, H. (2020). Percepciones y factores asociados a la salud bucal y la atención odontológica en el periodo perinatal en las mujeres y sus bebés. Odontología Sanmarquina, 23(3), 241-251. https://doi.org/10.15381/os.v23i3.18399. https://pesquisa.bvsalud.org/portal/resource/pt/biblio-1116689.

Costa, E. M., Azevedo, J. A. P. de, Martins, R. F. M., Rodrigues, V. P., Alves, C. M. C., Ribeiro, C. C. C., \& Thomaz, E. B. A. F. (2017). Níveis salivares de íons de ferro (Fe), marcadores séricos de anemia e atividade de cárie em gestantes. Revista Brasileira de Ginecologia e Obstetrícia,39(3), 94-101. http://dx.doi.org/10.1055/s-0037-1599217. https://www.scielo.br/scielo.php?script=sci_arttext\&pid=S0100-72032017000300094.

Costa, G. M. (2014). Protocolo de atenção à saúde bucal para gestantes na equipe da estratégia de saúde da família da "casa da comunidade serrinha" em Gouveia-MG. Trabalho de conclusão de curso apresentado ao curso de especialização em Atenção Básica em Saúde da Família, Universidade Federal de Minas Gerais, Lagoa Santa, MG. https://www.nescon.medicina.ufmg.br/biblioteca/imagem/4307.pdf.

Garbin, C. A. S., Sumida, D. H., Santos, R. R. dos, Chehoud, K. A., \& Moimaz, S. A. S. (2011). Saúde coletiva: promoção de saúde bucal na gravidez. Revista de Odontologia da UNESP, 40 (4), 161-165. http://hdl.handle.net/11449/133470.

Gesase, N., Miranda-Rius, J., Brunet-Llobet, L., Lahor-Soler, E., Mahande, M. J., \& Masenga, G. (2018). The association between periodontal disease and adverse pregnancy outcomes in Northern Tanzania: a cross-sectional study. African health sciences, 18(3), 601-611. https://doi.org/10.4314/ahs.v18i3.18. https://pubmed.ncbi.nlm.nih.gov/30602993/.

Gonçalves, K. F., Giordani, J. M. do A., Bidinotto, A. B., Ferla, A. A., Martins, A. B., \& Hilgert, J. B. (2020). Utilização de serviço de saúde bucal no prénatal na atenção primária à saúde: dados do PMAQ-AB. Ciência \& Saúde Coletiva, 25(2), 519-532. Epub 03 de feveiro de 2020. 10.1590/141381232020252.05342018. https://pesquisa.bvsalud.org/portal/resource/pt/biblio-1055808. 
Guidolini Martinelli, K., Belotti, L., Martins Poletto, Y., Theodoro dos Santos Neto, E., \& Emmerich Oliveira, A. (2020). Fatores associados ao cuidado de saúde bucal durante a gravidez. Arquivos Em Odontologia, 56. https://doi.org/10.7308/aodontol/2020.56.e16. https://periodicos.ufmg.br/index.php/arquivosemodontologia/article/view/16353.

Jacob, L. M. da S., Santos, A. P., Lopes, M. H. B. de M., \& Shimo, A. K. K. (2020). Perfil socioeconômico, demográfico e obstétrico de gestantes com Síndrome Hipertensiva de uma maternidade pública. Revista Gaúcha de Enfermagem, 41, e20190180. https://doi.org/10.1590/1983-1447.2020.20190180. https://www.scielo.br/scielo.php?pid=S1983-14472020000100410\&script=sci_arttext\&tlng=pt.

Konzen Júnior, D. J., Marmitt, L. P., \& Cesar, J. A. (2019). Não realização de consulta odontológica entre gestantes no extre mo sul do Brasil: um estudo de base populacional. Ciência \& Saúde Coletiva, 24(10), 3889-3896. https://doi.org/10.1590/1413-812320182410.31192017. https://www.scielo.br/scielo.php?script=sci_arttext\&pid=S1413-81232019001003889.

Lee, J. M., \& Shin, T. J. (2017). Use of local anesthetics for dental treatment during pregnancy; safety for parturient. Journal of dental anesthesia and pain medicine, 17(2), 81-90. https://doi.org/10.17245/jdapm.2017.17.2.81. https://www.ncbi.nlm.nih.gov/pmc/articles/PMC5564152/.

Lessa, I. B. (2013). Promoção à saúde bucal da gestante. Trabalho de conclusão de curso apresentado ao curso de especialização em Atenção Básica em Saúde da Família, Universidade Federal de Minas Gerais, Corinto, MG. https://www.nescon.medicina.ufmg.br/biblioteca/imagem/4268.pdf.

Lima, D. C. de, Saliba, N. A., Garbin, A. J. I., Fernandes, L. A, \& Garbin, C. A. S. (2011). A importância da saúde bucal na ótica de pacientes hospitalizados. Ciência \& Saúde Coletiva, 16(Suppl. 1), 1173-1180. http://dx.doi.org/10.1590/S1413-81232011000700049. https://www.scielo.br/scielo.php?script=sci_arttext\&pid=S1413-81232011000700049.

Lopes, F. F., Ribeiro, T. V., Fernandes, D. B., Calixto, N. R. de V., Alves, C. M. C., Pereira, A. L. A., \& Pereira, A. de F. V. (2016). Conhecimentos e práticas de saúde bucal de gestantes usuárias dos serviços de saúde em São Luís, Maranhão, 2007-2008. Epidemiologia e Serviços de Saúde,25(4), 819826. https://doi.org/10.5123/s1679-49742016000400015. https://www.scielo.br/scielo.php?pid=S2237-96222016000400819\&script=sci_abstract\&tlng=pt.

Lopes, I. K. R., Pessoa, D. M. da V., \& Macêdo, G. L. de. (2019). Autopercepção Do Pré-Natal Odontológico Pelas Gestantes De Uma Unidade Básica De Saúde. Revista Ciência Plural, 4(2), 60-72. https://periodicos.ufrn.br/rcp/article/view/16839.

Marla, V., Srii, R., Roy, D. K., \& Ajmera, H. (2018). The Importance of Oral Health during Pregnancy: A review. MedicalExpress, 5, https://www.scielo.br/scielo.php?script=sci_abstract\&pid=S2358-04292018000100201\&lng=en\&nrm=iso.

Martins, L. de O., Pinheiro, R. Di P. da S., Arantes, D. C., Nascimento, L. S. do, \& Santos Júnior, P. B. dos. (2013). Assistência odontológica à gestante: percepção do cirurgião-dentista. Dental care for pregnant woman: dental surgeon's perceptions. Revista Pan-Amazônica de Saúde,4(4), 11-18. http://dx.doi.org/10.5123/S2176-62232013000400002.

$62232013000400002 \& \operatorname{lng}=$ pt\&nrm=iso. http://scielo.iec.gov.br/scielo.php?script=sci_abstract\&pid=S2176-

Massoni, A. C. de L. T., Pereira, R. B., Nóbrega, D. R. M., Costa, L. E. D., Fernandes, J. M. F. de A., \& Rosenblatt, A. (2015). Assessment of pregnant, primiparous and postpartum women's knowledge about dental caries. RGO - Revista Gaúcha de Odontologia, 63(2), 145-152. https://doi.org/10.1590/1981863720150002000022808. https://www.scielo.br/scielo.php?script=sci_arttext\&pid=S1981-86372015000200145.

Mattos, B., \& Davoglio, R. (2015). Saúde bucal: a voz da gestante. Revista Da Faculdade De Odontologia - UPF, 20(3). https://doi.org/10.5335/rfo.v20i3.4891. http://www.seer.upf.br/index.php/rfo/article/view/4891.

Moimaz, S. A., Rós, D. de T., Saliba, T. A., Garbim, C. A. S. (2017). Aspectos da saúde geral e bucal de gestantes de alto risco: revisão de literatura. J. Health Sci. Inst, 35(3), 223-230. https://pesquisa.bvsalud.org/portal/resource/pt/biblio-882763.

Moimaz, S. A. S., Rovida, T. A. S., Garbin, C. A. S., Santos, A. da S., Saliba, N. A. (2015). Saúde bucal e o emprego de medidas preventivas por pacientes gestantes. J. Health Sci. Inst, 33(4), 328-332. https://pesquisa.bvsalud.org/portal/resource/pt/biblio-2138.

Nunes Neto, R.A., \& Frutuoso, M.F.P. (2018). Oral health and the care of pregnant women: workshops as a strategy to problematize practices in basic health care in residents living in the peripheral areas of the hills in the city of Santos. RGO, Rev Gaúch Odontol. 66(4), 305-316. http://dx.doi. org/10.1590/1981863720180004000033504. https://www.scielo.br/scielo.php?pid=S1981-86372018000400305\&script=sci_arttext.

Oliveira, E. C. de, Lopes, J. M. O., Santos, P. C. F., Magalhães, S. R. (2014). Atendimento odontológico a gestantes: a importância do conhecimento da saúde bucal. Revista de Iniciação Científica da Universidade Vale do Rio Verde 4(1), 11-23. http://periodicos.unincor.br/index.php/iniciacaocientifica/article/view/1550.

Pacheco, K. T. dos S., Sakugawa, K. O., Martinelli, K. G., Esposti, C. D. D., Pacheco Filho, A. C., Garbin, C. A. S., Garbin, A. J. I., \& Santos Neto, E. T. (2020). Saúde bucal e qualidade de vida de gestantes: a influência de fatores sociais e demográficos. Ciência \& Saúde Coletiva, 25(6), 2315-2324. https://doi.org/10.1590/1413-81232020256.24002018. https://www.scielo.br/scielo.php?pid=S1413-81232020000602315\&script=sci_arttext.

Pereira, A. L. (2010). Influência da condição de saúde bucal na qualidade de vida dos indivíduos. Trabalho de conclusão de curso apresentado ao curso de especialização em Atenção Básica em Saúde da Família, Universidade Federal de Minas Gerais, Campos Gerais, MG. https://www.nescon.medicina.ufmg.br/biblioteca/imagem/0986.pdf.

Piccirillo, N. P. (2012). O atendimento odontológico da gestante: fundamentos e possibilidades. Trabalho de conclusão de curso apresentado ao curso de especialização em Atenção Básica em Saúde da Família, Universidade Federal de Minas Gerais, São Sebastião do Paraíso. https://www.nescon.medicina.ufmg.br/biblioteca/imagem/Atendimento_odontol\%C3\%B3gico_gestante.pdf.

Reis, D. M., Pitta, D. R., Ferreira, H. M. B., Jesus, M. C. P. de, Moraes, M. E. L. de, \& Soares, M. G. (2010). Educação em saúde como estratégia de promoção de saúde bucal em gestantes. Ciência \& Saúde Coletiva, 15(1), 269-276. https://doi.org/10.1590/S1413-81232010000100032. https://www.scielo.br/scielo.php?script=sci_abstract\&pid=S1413-81232010000100032\&lng=es\&nrm=iso\&tlng=pt. 
Research, Society and Development, v. 10, n. 1, e56810112234, 2021

(CC BY 4.0) | ISSN 2525-3409 | DOI: http://dx.doi.org/10.33448/rsd-v10i1.12234

Rocha, J. S., Arima, L., Chibinski, A. C., Werneck, R. I., Moysés, S. J., \& Baldani, M. H. (2018). Barriers and facilitators to dental care during pregnancy: a systematic review and meta-synthesis of qualitative studies. Cadernos de Saúde Pública, 34(8), e00130817. 2018.https://doi.org/10.1590/0102-311x00130817. https://www.scielo.br/scielo.php?script=sci_arttext\&pid=S0102-311X2018000802001.

Rodrigues, F., Mármora, B., Carrion, S. J., Rego, A. E. C., Pospich, F. S. (2017). Anestesia local em gestantes na odontologia contemporânea. Journal Health NPEPS, 2(1), 254-271. https://periodicos.unemat.br/index.php/jhnpeps/article/view/1835/0.

Rosa, M. I. da, Pires, P. D. S., Medeiros, L. R., Edelweiss, M. I., \& Martínez-Mesa, J. (2012). Periodontal disease treatment and risk of preterm birth: a systematic review and meta-analysis. Cadernos de Saúde Pública,28(10), 1823-1833. https://doi.org/10.1590/S0102-311X2012001000002. https://www.scielo.br/scielo.php?pid=S0102-311X2012001000002\&script=sci_arttext\#: :text=The\%20meta\%2Danalysis\%20of\%20t he,clear\%20evidence\%2 0of\%20publication\%20bias.

Sá de Lira, A. de L., Silva, N. R. F. da, Caetano, V. da S., Araújo Junior, A. G. da, \& Portela, I. J. Z. (2019). Prevalence and etiological factors of Piogenic Granuloma in gestants. Brazilian Dental Science. 22(4), 443-449. https://pesquisa.bvsalud.org/portal/resource/pt/biblio-1024304.

Saliba, T. A., Custódio, L. B. de M., Saliba, N. A., \& Moimaz, S. A. S. (2019). Dental prenatal care in pregnancy. RGO - Revista Gaúcha de Odontologia, 67, e20190061. https://doi.org/10.1590/1981-863720190006120180003 https://www.scielo.br/scielo.php?script=sci_arttext\&pid=S1981-86372019000100329.

Silva, C. C. da, Savian, C. M., Prevedello, B. P., Zamberlan, C., Dalpian, D. M., \& Santos, B. Z. dos. (2020). Acesso e utilização de serviços odontológicos por gestantes: revisão integrativa de literatura. Ciência \& Saúde Coletiva, 25(3), 827-835. https://doi.org/10.1590/1413-81232020253.01192018. https://www.scielo.br/scielo.php?script=sci_arttext\&pid=S1413-81232020000300827.

Sousa, L. L. A. de, Cagnani, A., Barros, A. M. de S., Zanin, L., \& Flório, F. M. (2016). Pregnant women's oral health: knowledge, practices and their relationship with periodontal disease. RGO - Revista Gaúcha de Odontologia, 64(2), 154-163. https://dx.doi.org/10.1590/1981-863720160002000053036. https://www.scielo.br/scielo.php?script=sci_arttext\&pid=S1981-86372016000200154.

Teshome, A., \& Yitayeh, A. (2016). Relationship between periodontal disease and preterm low birth weight: systematic review. The Pan African medical journal, 24, 215. https://doi.org/10.11604/pamj.2016.24.215.8727. https://pubmed.ncbi.nlm.nih.gov/27800070/.

Trujillo Saínz, Z. de la C., Paz Paula, C. M., Hernández Acosta, Y., \& Henriquez Trujillo, D. (2019). Salud bucal y diabetes gestacional en el Centro Provincial de Atención al Diabético. Revista de Ciencias Médicas de Pinar del Río,23(4), 513-522. http://scielo.sld.cu/scielo.php?script=sci_arttext\&pid=S1561-31942019000400513. 